\title{
Correction to: Association of plasma potassium with mortality and end-stage kidney disease in patients with chronic kidney disease under nephrologist care - The NephroTest study
}

Sandra Wagner ${ }^{1,2}$, Marie Metzger ${ }^{1}$, Martin Flamant ${ }^{3}$, Pascal Houillier ${ }^{4,5}$, Jean-Philippe Haymann ${ }^{6}$, François Vrtovsnik ${ }^{3}$, Eric Thervet ${ }^{5,7}$, Jean-Jacques Boffa ${ }^{6}$, Ziad A. Massy ${ }^{1,2,8}$, Bénédicte Stengel ${ }^{1,2^{*}}$,

Patrick Rossignol ${ }^{2,9}$ and for the NephroTest Study group

In the original version of this article [1], published on 12 September 2017, the explanation of ${ }^{\mathrm{a}}$ and ${ }^{\mathrm{b}}$ in the footnote of Table 2 were switched during typesetting. In this Correction Table 2, the incorrect and correct version of its footnote are shown. The affected part of the footnote is marked in italics. The original publication of this article has been corrected.

\section{Author details}

'CESP, Inserm U1018, Univ Paris-Saclay, Univ Paris-Sud, UVSQ, Villejuif, France. ${ }^{2}$ FCRIN INI-CRCT, Paris, France. ${ }^{3}$ Bichat Hospital, Paris, France. ${ }^{4}$ INSERM U1138, Paris, France. ${ }^{5} \mathrm{HEGP}$, Paris, France. ${ }^{6}$ Tenon Hospital, Paris, France. ${ }^{7}$ INSERM, UMRS970, Boulogne-Billancourt, France. ${ }^{8}$ Ambroise Paré Hospital, Boulogne-Billancourt, France. ${ }^{9}$ INSERM CIC 1433, Nancy CHRU and University of Lorraine, Nancy, France.

Received: 22 September 2017 Accepted: 22 September 2017 Published online: 06 October 2017

\section{Reference}

1. Wagner et al. Association of plasma potassium with mortality and end-

stage kidney disease in patients with chronic kidney disease under nephrologist care - The NephroTest study (2017) 18:295 DOI 10.1186/ s12882-017-0710-7

\footnotetext{
* Correspondence: benedicte.stengel@inserm.fr

${ }^{1}$ CESP, Inserm U1018, Univ Paris-Saclay, Univ Paris-Sud, UVSQ, Villejuif, France

${ }^{2}$ FCRIN INI-CRCT, Paris, France
} 
Originally Table 2 and its footnote were published as followed:

Table 2 Odds ratios of low or high plasma potassium associated with baseline patient characteristics - Multinomial logistic regression using patients with plasma potassium of $4-5 \mathrm{mmol} / \mathrm{L}$ as the reference group

\begin{tabular}{|c|c|c|c|}
\hline & & \multicolumn{2}{|c|}{ Plasma potassium (mmol/L) } \\
\hline & & $<4$ & $>5$ \\
\hline Age (per year) & & $0.99(0.98-1.00)$ & $0.98(0.96-0.99)$ \\
\hline Women vs men & & $1.49(1.16-1.90)$ & $0.47(0.30-0.72)$ \\
\hline Sub-Saharan vs other ethnicity & & $1.35(0.99-1.83)$ & $1.15(0.66-1.99)$ \\
\hline \multicolumn{4}{|l|}{$\mathrm{mGFR}\left(\mathrm{ml} / \mathrm{min} / 1.73 \mathrm{~m}^{2}\right)$} \\
\hline & $<15$ & $0.11(0.05-0.23)$ & $29.65(10.87-80.88)$ \\
\hline & $15-30$ & $0.26(0.18-0.38)$ & $13.58(5.71-32.3)$ \\
\hline & $30-45$ & $0.47(0.34-0.63)$ & $5.70(2.42-13.45)$ \\
\hline & $45-60$ & $0.67(0.49-0.90)$ & $2.70(1.07-6.85)$ \\
\hline & $>60$ & 1 & 1 \\
\hline \multicolumn{4}{|l|}{ BMl } \\
\hline & $<19$ & $1.46(0.83-2.59)$ & $1.49(0.65-3.43)$ \\
\hline & $19-25$ & 1 & 1 \\
\hline & $25-30$ & $0.85(0.66-1.10)$ & $0.78(0.51-1.20)$ \\
\hline & $>30$ & $0.78(0.57-1.07)$ & $1.05(0.65-1.73)$ \\
\hline \multicolumn{4}{|l|}{ Smoking status } \\
\hline & Never smoked & 1 & 1 \\
\hline & Former smoker & $0.85(0.66-1.11)$ & $1.02(0.67-1.54)$ \\
\hline & Active smoker & $0.76(0.54-1.06)$ & $1.66(1.03-2.67)$ \\
\hline Mean blood pressure (per mmHg) & & $1.01(1.00-1.02)$ & $1.01(0.99-1.02)$ \\
\hline Cardio-vascular history & & $0.63(0.46-0.88)$ & $0.81(0.51-1.28)$ \\
\hline \multicolumn{4}{|l|}{ ACR (mg/mmol) } \\
\hline & $<3$ & 1 & 1 \\
\hline & $3-30$ & $1.07(0.82-1.40)$ & $1.25(0.76-2.08)$ \\
\hline & $>30$ & $0.80(0.59-1.10)$ & $1.13(0.67-1.90)$ \\
\hline Diabetes & & $0.86(0.66-1.13)$ & $1.56(1.04-2.34)$ \\
\hline Urine potassium & & $0.99(0.99-1.00)$ & $1.01(1.00-1.01)$ \\
\hline \multicolumn{4}{|l|}{ Serum albumin } \\
\hline & $\geq 35$ & 1 & 1 \\
\hline & $<35$ & $1.23(0.87-1.74)$ & $1.23(0.76-1.98)$ \\
\hline Serum potassium increasing drugs ${ }^{a}$ & & $0.58(0.44-0.78)$ & $2.50(1.17-5.35)$ \\
\hline Serum potassium-lowering drugs ${ }^{b}$ & & $1.70(1.33-2.17)$ & $1.01(0.69-1.49)$ \\
\hline
\end{tabular}

aloop or thiazide diuretic, kayexalate or bicarbonates ${ }^{\mathrm{b}} \mathrm{ACEi}$ or ARBs or potassium-sparing diuretics

BMI body mass index, CVD cardiovascular disease, mGFR measured GFR, ACR, ratio of urinary albumin to creatinine

The analyses was adjusted for center 
The correct version of Table 2 and its footnote is:

Table 2 Odds ratios of low or high plasma potassium associated with baseline patient characteristics - Multinomial logistic regression using patients with plasma potassium of $4-5 \mathrm{mmol} / \mathrm{L}$ as the reference group

\begin{tabular}{|c|c|c|c|}
\hline & & \multicolumn{2}{|c|}{ Plasma potassium (mmol/L) } \\
\hline & & $<4$ & $>5$ \\
\hline Age (per year) & & $0.99(0.98-1.00)$ & $0.98(0.96-0.99)$ \\
\hline Women vs men & & $1.49(1.16-1.90)$ & $0.47(0.30-0.72)$ \\
\hline Sub-Saharan vs other ethnicity & & $1.35(0.99-1.83)$ & $1.15(0.66-1.99)$ \\
\hline \multicolumn{4}{|l|}{$\operatorname{mGFR}\left(\mathrm{ml} / \mathrm{min} / 1.73 \mathrm{~m}^{2}\right)$} \\
\hline & $<15$ & $0.11(0.05-0.23)$ & $29.65(10.87-80.88)$ \\
\hline & $15-30$ & $0.26(0.18-0.38)$ & $13.58(5.71-32.3)$ \\
\hline & $30-45$ & $0.47(0.34-0.63)$ & $5.70(2.42-13.45)$ \\
\hline & $45-60$ & $0.67(0.49-0.90)$ & $2.70(1.07-6.85)$ \\
\hline & $>60$ & 1 & 1 \\
\hline \multicolumn{4}{|l|}{ BMl } \\
\hline & $<19$ & $1.46(0.83-2.59)$ & $1.49(0.65-3.43)$ \\
\hline & $19-25$ & 1 & 1 \\
\hline & $25-30$ & $0.85(0.66-1.10)$ & $0.78(0.51-1.20)$ \\
\hline & $>30$ & $0.78(0.57-1.07)$ & $1.05(0.65-1.73)$ \\
\hline \multicolumn{4}{|l|}{ Smoking status } \\
\hline & Never smoked & 1 & 1 \\
\hline & Former smoker & $0.85(0.66-1.11)$ & $1.02(0.67-1.54)$ \\
\hline & Active smoker & $0.76(0.54-1.06)$ & $1.66(1.03-2.67)$ \\
\hline Mean blood pressure (per mmHg) & & $1.01(1.00-1.02)$ & $1.01(0.99-1.02)$ \\
\hline Cardio-vascular history & & $0.63(0.46-0.88)$ & $0.81(0.51-1.28)$ \\
\hline \multicolumn{4}{|l|}{ ACR (mg/mmol) } \\
\hline & $<3$ & 1 & 1 \\
\hline & $3-30$ & $1.07(0.82-1.40)$ & $1.25(0.76-2.08)$ \\
\hline & $>30$ & $0.80(0.59-1.10)$ & $1.13(0.67-1.90)$ \\
\hline Diabetes & & $0.86(0.66-1.13)$ & $1.56(1.04-2.34)$ \\
\hline Urine potassium & & $0.99(0.99-1.00)$ & $1.01(1.00-1.01)$ \\
\hline \multicolumn{4}{|l|}{ Serum albumin } \\
\hline & $\geq 35$ & 1 & 1 \\
\hline & $<35$ & $1.23(0.87-1.74)$ & $1.23(0.76-1.98)$ \\
\hline Serum potassium increasing drugs $^{a}$ & & $0.58(0.44-0.78)$ & $2.50(1.17-5.35)$ \\
\hline Serum potassium-lowering drugs ${ }^{b}$ & & $1.70(1.33-2.17)$ & $1.01(0.69-1.49)$ \\
\hline
\end{tabular}

${ }^{a}$ ACEi or ARBs or potassium-sparing diuretics ${ }^{\mathrm{b}}$ loop or thiazide diuretic, kayexalate or bicarbonates

BMI, body mass index, CVD, cardiovascular disease, mGFR, measured GFR, ACR, ratio of urinary albumin to creatinine

The analyses was adjusted for center 